\title{
A Case of Reversible Cerebral Vasoconstriction Syndrome: Postpartum Cerebral Angiopathy in a 32-Year-Old Primigravid Treated with Intravenous Immunoglobulin
}

\author{
John Angelo Luigi S. Perez Manilen E. Dueñas Senna S. Lim \\ Geraldine Siena L. Mariano Jose C. Navarro \\ Institute for Neurosciences, St. Luke's Medical Center, Quezon City, Philippines
}

\section{Keywords}

Reversible cerebral vasoconstriction syndrome $\cdot$ Postpartum cerebral angiopathy $\cdot$ Cerebral infarction

\begin{abstract}
This is a case of a 32-year-old primigravid who developed sudden severe headache on the 7th day postpartum associated with focal neurologic deficits and altered sensorium. She had a GCS score of 6, anisocoric pupils and an NIHSS score of 31. Cranial MRI with MRA showed multifocal hyperacute to acute infarcts on the left occipital lobe, left thalamus, and midbrain which was more prominent on the right. Due to clinical deterioration, a repeat Cranial MRI with MRA was done and showed progression of infarcts involving both thalami and right pons with interval appearance of contour irregularities in the proximal anterior cerebral, posterior cerebral, basilar and internal carotid arteries. Serial transcranial Doppler showed significant distal right middle cerebral artery vasospasm. She was managed as a case of reversible cerebral vasoconstriction syndrome, associated with postpartum cerebral angiopathy. Intravenous pulse methylprednisolone was started subsequently IVIG was initiated. Intravenous immunoglobulin was given for 5 days. The patient gradually improved, underwent rehabilitation therapy, and was discharged stable after 6 weeks.
\end{abstract}


Perez et al.: Postpartum Cerebral Angiopathy in a 32-Year-Old Primigravid Treated with IVIg

\section{Introduction}

It has been shown that stroke in young adults is more common in females than in males, with poorer outcomes in females. Additional risk factors in women include pregnancy, use of oral contraceptives, and hormonal therapy [1]. Stroke in pregnancy is relatively rare; however, certain risk factors may be associated with stroke in females during pregnancy these include age more than 35 years, black ethnicity, hypertension, heart disease, smoking, diabetes, lupus, sickle cell disease, migraine headaches, alcohol and substance abuse, caesarean delivery, fluid and electrolyte disorders, thrombophilia, multiple gestations, greater parity, and postpartum infection $[1,2]$. Furthermore, specific stroke syndromes in pregnancy include pre-eclampsia and eclampsia, subarachnoid hemorrhage secondary to aneurysmal rupture, cerebral venous thrombosis, paradoxical embolism, peripartum cardiomyopathy and postpartum cerebral angiopathy (PCA) (as the present case) [1].

\section{Case Report}

The patient is a 32-year-old primigravid, diagnosed case of diet-controlled gestational diabetes, who gave birth to a live, full-term, healthy boy via spontaneous vaginal delivery under epidural anesthesia. The pregnancy and postoperative courses were unremarkable, and no complications were observed. She was discharged well after the 3rd day postpartum.

On the 7th day postpartum, the patient experienced sudden severe throbbing headache, occipital in location, with associated elevations in blood pressure up to $170 / 100 \mathrm{~mm} \mathrm{Hg}$. This was persistent throughout the day, with no associated nausea, vomiting, or focal neurologic deficit. No medications were taken, and the headache became persistent with minimal improvement with rest. On the 9th day postpartum, she was seen by her husband to be unresponsive and unarousable from sleep. She was immediately brought to the emergency room for evaluation.

The patient arrived at the Emergency Department unresponsive with a Glasgow Coma Scale (GCS) score of $6\left(E_{1} V_{1} M_{4}\right)$ with no eye-opening, no verbal output, and was only able to withdraw her extremities to painful stimulation. Vital signs were the following: BP 180/100 $\mathrm{mm} \mathrm{Hg}$, HR $40 \mathrm{bpm}$, regular, RR $10 \mathrm{cpm}$ and $\mathrm{O}_{2}$ saturation was $99 \%$ at room air. Breath sounds were clear. Her abdomen was soft with no guarding, and her uterus was well contracted with no signs of gross vaginal bleeding. She had grade 1 bipedal edema, but peripheral pulses were full and equal. Upon immediate referral to Neurology and Stroke Service, neurologic examination showed extensor posturing on the right and localization to pain on the left. She was anisocoric. The right pupil measured $6 \mathrm{~mm}$ while the left pupil measured $3 \mathrm{~mm}$. Both were nonreactive to light and had no visual threat. Oculocephalic, corneal, and gag reflexes were intact. There was bilateral toe extensor response. Her NIHSS score was 31 and indicated a severe stroke. Rapid sequence intubation was done, and she was hooked to a mechanical ventilator.

Cranial MRI with MRA showed restricted diffusion in the left occipital lobe, left thalamus, and the midline midbrain but was more prominent on the right. The ADC map showed corresponding signal drops. These MRI findings were consistent with hyperacute to acute ischemic infarction (see Fig. 1).

The patient was initially managed as a case of acute posterior circulation stroke probably secondary to cardioembolism versus hypercoagulable state. Intravenous heparin infusion titrated to maintain a partial thromboplastin time of 1.5-2.3 times the baseline was started. There was initial improvement of sensorium to GCS $10\left(\mathrm{E}_{3} \mathrm{~V}_{1} \mathrm{M}_{6}\right)$; however, on the $3 r d$ hospital day, there was deterioration in sensorium to GCS $6\left(E_{1} M_{1} V_{4}\right)$. Repeat cranial

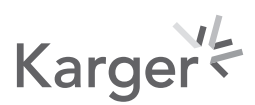




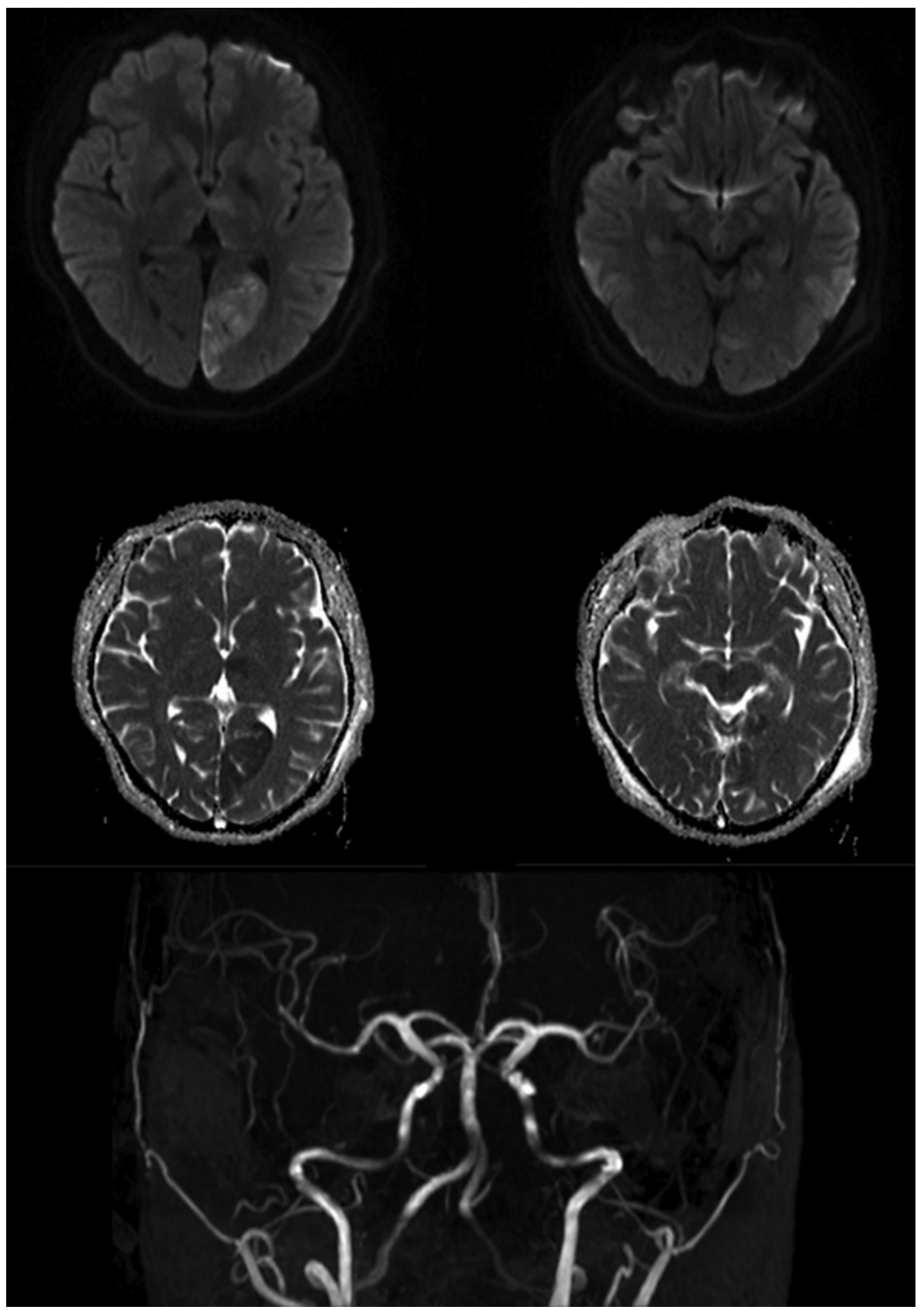

Fig. 1. Noncontrast enhanced cranial MRI showing restricted diffusion with corresponding signal loss on ADC in the left thalamus, left occipital lobe, and midline midbrain. TOF angiography was unremarkable.

MRI showed progression of infarcts now involving both thalami and the right side of the pons. On TOF angiography, there was interval appearance of contour irregularities in the proximal anterior cerebral, posterior cerebral, basilar, and internal carotid arteries (see Fig. 2).

With interval findings of narrowing of intracranial arteries, she was then managed as a case of reversible cerebral vasoconstriction syndrome (RCVS), associated with postpartum cerebral angiopathy (PCA). Heparinization was discontinued, and the patient was started on intravenous pulse methylprednisolone $250 \mathrm{mg}$ IV every $6 \mathrm{~h}$ and nimodipine 60 


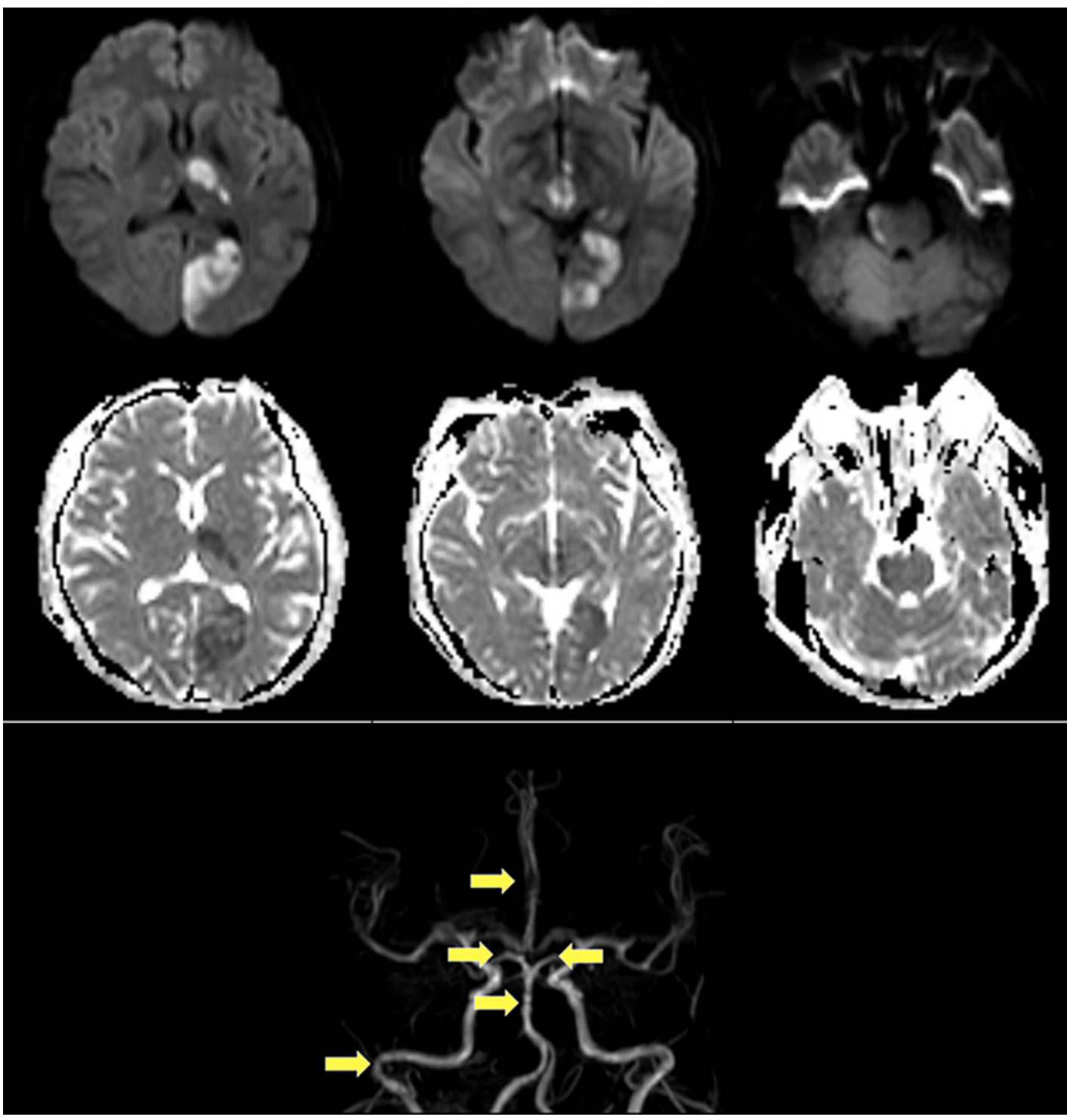

Fig. 2. Follow-up noncontrast enhanced cranial MRI showing progression of infarcts now involving bilateral thalami, upper midbrain, and the right circumferential pons. TOF angiography demonstrated narrowing of the proximal anterior cerebral, posterior cerebral, basilar, and internal carotid arteries (yellow arrows).

mg every $4 \mathrm{~h}$. Aspirin $80 \mathrm{mg}$ once daily and atorvastatin $40 \mathrm{mg}$ once daily were also started. There was subsequent improvement to GCS $11\left(\mathrm{E}_{4} \mathrm{~V}_{1} \mathrm{M}_{6}\right)$ the following day. However, on the 6th hospital day, there was further deterioration to GCS $7\left(E_{1} V_{1} M_{5}\right)$. Intravenous immunoglobulin $30 \mathrm{~g}$ per day $(\sim 0.4 \mathrm{~g} / \mathrm{kg} /$ day $)$ was started and was given for 5 days.

A series of diagnostic tests were done to investigate the possible disease mechanism. Work-up for autoimmune, connective tissue disease, and stroke in the young was negative (see Table 1). While both ESR and CRP were slightly elevated, they were not considered significant as they are nonspecific and may be elevated in severe acute illness.

In addition to serial MR angiography, transcranial Doppler was performed and noted significant distal right MCA vasospasm. This was monitored throughout the patient's admission (see Fig. 3; Table 2). 
Table 1. Summary of diagnostics for stroke in the young, autoimmune, and connective tissue disease

\begin{tabular}{lll}
\hline Test & Value & Reference value \\
\hline Erythrocyte sedimentation rate & 21 & $0-20 \mathrm{~mm} / \mathrm{h}$ \\
C-reactive protein & 12 & $<6 \mathrm{mg} / \mathrm{L}$ \\
Antinuclear antibody & $1: 40$ speckled & \\
Complement 3 & 114 & $79-152 \mathrm{mg} / \mathrm{dL}$ \\
Antithrombin III & 82 & $82 \%$ \\
Factor V leiden & 0.9 & $>0.8$ \\
Protein C & 114 & $70-140 \%$ \\
Protein S & 90 & $60-140 \%$ \\
Lupus anticoagulant & 1.1 & \\
\hline
\end{tabular}

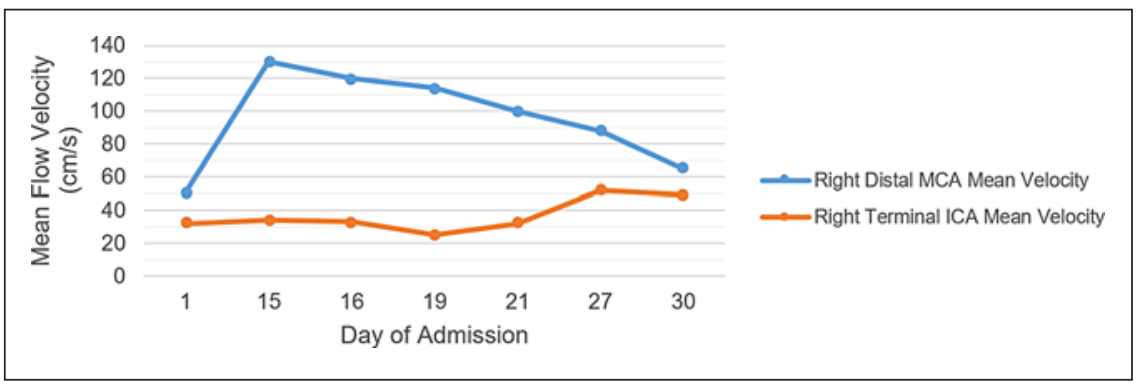

Fig. 3. Summary of mean flow velocities $(\mathrm{cm} / \mathrm{s})$ of the right distal MCA, ipsilateral ICA.

The patient gradually improved over the following days. Intravenous methylprednisolone was shifted to oral prednisone after 4 days with gradual tapering over 8 weeks while oral nimodipine was continued. Wakefulness became spontaneous and sustained and she was now able to follow commands. She was eventually extubated on the 17th hospital day. Physical rehabilitation and speech therapy were done daily.

After 4 weeks from the onset of symptoms, gradual improvement was noted and she became fully awake, alert and was able to hold simple and short conversations. Mood was improved and she was noted to be more interactive with the medical staff and visitors. Pupils remained anisocoric and nonreactive to light with limited vertical gaze. There was still generalized weakness, but she was able to move all her extremities spontaneously and purposefully. The patient was discharged stable and well after 6 weeks.

\section{Discussion}

RCVS, particularly PCA was the working diagnosis for this case and the patient was managed as such. This was supported by her initial presentation of acute headache followed by neurological signs and symptoms and further deterioration during her clinical course. These were correlated with neuroimaging findings of multiple cerebral infarcts of different vascular territories and interval narrowing or constriction of cerebral vessels by serial Cranial MRI and MRA. Serial transcranial Doppler studies were also done. Neurologic signs and symptoms were gradually improving, suggesting reversibility, over weeks with supportive measures and therapeutic management with intravenous immunoglobulin. 
Table 2. Summary of pulsatility indices

\begin{tabular}{lllllll}
\hline Artery & \multicolumn{7}{l}{ Days post-ictus, $n$} & & & \\
\cline { 2 - 7 } & D1 & D15 & D19 & D21 & D27 & D30 \\
\hline Right MCA & 0.94 & 1.13 & 1.05 & 0.88 & 1.28 & 1.02 \\
Left MCA & 1.02 & 0.97 & 0.67 & 0.78 & 0.8 & 0.77 \\
Right ACA & 0.73 & 0.91 & 1.03 & 0.99 & 1.02 & 0.72 \\
Left ACA & 0.92 & 1.17 & 0.82 & 0.72 & 0.73 & 1 \\
Right PCA & 1.14 & 1.17 & 0.81 & 1.11 & 0.77 & 0.71 \\
Left PCA & 0.84 & 0.83 & 0.62 & 0.61 & 0.89 & 0.77 \\
Right ICA & 1.13 & 1.04 & 0.87 & 1.03 & 0.66 & 0.91 \\
Left ICA & 1.1 & 0.82 & 0.88 & 1.3 & 0.9 & 0.86 \\
Right carotid siphon & 0.76 & 0.83 & 0.71 & 0.82 & 1 & 0.86 \\
Left carotid siphon & 1.05 & 1.1 & 1.09 & 0.82 & 0.95 & 0.7 \\
Right ophthalmic & 1.38 & 1.4 & 1.83 & 1.62 & 1.56 & 1.28 \\
Left ophthalmic & 1.36 & 1.5 & 1.47 & 1.48 & 1.57 & 1.42 \\
Right vertebral & 0.75 & 0.87 & 0.74 & 0.92 & 0.92 & 0.56 \\
Left vertebral & 1.22 & 0.73 & 0.88 & 1.1 & 0.97 & 0.84 \\
Basilar & 1.14 & 0.92 & 0.79 & 0.7 & 0.94 & 0.81 \\
\hline
\end{tabular}

PCA is under the category of disorders termed RCVS. These are characterized by reversible multifocal vasoconstriction of the cerebral arteries and occur in a variety of clinical settings including pregnancy and the puerperium, drug exposure, migraine, mechanical trauma, hypercalcemia, and idiopathic causes with no obvious precipitating factor [1]. PCA also shares similarities with the closely related posterior reversible encephalopathy syndrome (PRES). Both conditions present with bilateral posterior-dominant lesions on MRI; however, the main differentiators between the two are that PCA presents with restricted diffusion and signal drops on apparent diffusion coefficient maps. On the other hand, PRES presents with T2 and FLAIR hyperintensities. The significant implication here is that the pathology of PCA involves cerebral infarction, while PRES involves cerebral edema brought about by cerebral endothelial dysfunction and leakage [2]. Clinical characteristics greatly overlap as well as both conditions may present with alterations in sensorium, seizures, headache, and focal neurologic deficits. Neuroimaging then plays an important role in differentiating them as their management strategies differ.

PCA is characterized by prolonged, but reversible vasoconstriction of the cerebral arteries. It is associated with acute onset, severe, thunderclap, recurrent headaches, with or without altered consciousness and transient or permanent neurologic signs and symptoms $[3,4]$. Most patients have a history of uncomplicated pregnancy and normal labor and delivery, followed within days to a few weeks by the aforementioned symptoms $[1,3]$.

The cranial MRI in patients with PCA may show areas of T2/FLAIR hyperintensity in any location, but especially in watershed areas between vascular territories. Radiological features include multifocal segmental narrowing of the cerebral arteries, particularly the large- and medium-sized vessels, with reversibility and complete resolution 4-6 weeks later. Arterial abnormalities are better visualized with CT angiography and conventional invasive catheter angiography. The MRI and MRA features may normalize with time, although extensive infarction may develop [1-4]. 
PCA has overlapping clinical and angiographic features with cerebral vasculitis. Cerebrospinal fluid analysis may be helpful during diagnostic uncertainty, and this is often unremarkable in PCA. Some cases may require brain biopsy with therapeutic and prognostic implications [1].

Diagnostic criteria of RCVS include (1) intense, acute headache, with or without additional neurological signs or symptoms; (2) catheter, CT, or MRI angiography demonstrating segmental cerebral artery vasoconstriction; (3) no evidence for aneurysmal SAH (4) reversibility of angiographic abnormalities within 12 weeks of onset; and (5) CSF analysis normal or close to normal (protein level $<80 \mathrm{mg} / \mathrm{dL}$, leukocytes $<10 / \mathrm{mm}^{3}$, normal glucose level) [5]. If death occurs before completion of work-up, then autopsy has to rule out vasculitis, intracranial atherosclerosis, and aneurysmal SAH, which can also manifest with headache and stroke.

The pathophysiology of PCA remains unclear. A disturbance in the control of vascular tone is likely to play a central part, and factors known to be responsible for SAH-related vasospasm may be responsible. Some cases of PCA have occurred in association with the use of vasospastic drugs such as ergonovine and bromocriptine during pregnancy, as previously mentioned $[1,3]$.

In terms of treatment, no clinical trials have been done to investigate the suitable management of this disorder. Therapies have been guided by observational data. All patients need symptomatic and supportive treatment which may include analgesics, antiepileptic drugs for seizures, blood pressure monitoring, and admission to the intensive care unit in severe cases. Calcium-channel blockers (nimodipine or verapamil), glucocorticoids, and magnesium sulfate, have been proposed. In the most severe cases, milrinone, nimodipine, and epoprostenol administered intra-arterially, and balloon angioplasty with variable success have been used. These are delicate and high-risk interventions and should be restricted to patients who show clear signs of clinical progression $[1,5]$. There have also been reports of immunoglobulin therapy for severe cases [6].

\section{Acknowledgement}

The authors would like to acknowledge Drs. Joven R. Cuanang, MD; Joyce Kristine L. Porto, MD, and Kathleen Jay L. Luspian, MD for their input in the management of this case and critique of the report.

\section{Statement of Ethics}

This research has complied with the guidelines for human studies in accordance with the World Medical Association of Helsinki. The subject's family has given written informed consent to publish their case. This report has been approved by the Institutional Review Board.

\section{Conflict of Interest Statement}

The authors have no conflicts of interest to declare.

\section{Funding Sources}

This case report did not receive any specific grant from funding agencies in the public, commercial, or not-for-profit sectors.

\section{Karger'}




\section{Author Contributions}

The authors contributed to the following aspects of this paper: conception and design: Drs. Perez, Dueñas, Lim, Mariano, Navarro. Data collection: Drs. Perez, Dueñas. Data analysis and interpretation: Drs. Perez, Dueñas, Lim, Mariano, Navarro. Article drafting: Drs. Perez, Dueñas. Critical revisions: Drs. Perez, Dueñas, Lim, Mariano, Navarro. Final approval: Drs. Perez, Dueñas, Lim, Mariano, Navarro.

\section{Data Availability Statement}

The datasets used and/or analyzed during the current case report are available from the corresponding author on reasonable request.

\section{References}

1 Treadwell SD, Thanvi B, Robinson TG. Stroke in pregnancy and the puerperium. Postgrad Med J. 2008;84:238-45.

2 Fugate JE, Claassen DO, Cloft HJ, Kallmes DF, Kozak OS, Rabinstein AA. Posterior reversible encephalopathy syndrome: associated clinical and radiologic findings. Mayo Clin Proc. 2010;85(5):427-32.

3 Del Zotto E, Giossi A, Volonghi I, Costa P, Padovani A, Pezzini A. Ischemic stroke during pregnancy and puerperium. Stroke Res Treat. 2011;2011:606780. Article ID 606780.

4 Tang SC, Jeng JS. Management of stroke in pregnancy and the puerperium. Expert Rev Neurother. 2010;10(2):205.

5 Santos L, Azevedo E. Reversible cerebral vasoconstriction syndrome: a narrative revision of the literature. Porto Biomed J. 2016;1(2):65-71.

6 Samaniego EA, Dabus G, Generoso GM, Tari-Capone F, Fuentes K, Linfante I. Postpartum cerebral angiopathy treated with intra-arterial nicardipine and intravenous immunoglobulin. J Neurointerv Surg. 2013;5:e12.

\section{Karger' ${ }^{\prime}$}

